\title{
MODULUS OF SMOOTHNESS AND THEOREMS CONCERNING APPROXIMATION ON COMPACT GROUPS
}

\author{
H. VAEZI and S. F. RZAEV
}

Received 3 April 2002

\begin{abstract}
We consider the generalized shift operator defined by $\left(\mathrm{Sh}_{u} f\right)(g)=\int_{G} f\left(t u t^{-1} g\right) d t$ on a compact group $G$, and by using this operator, we define "spherical" modulus of smoothness. So, we prove Stechkin and Jackson-type theorems.
\end{abstract}

2000 Mathematics Subject Classification: 42C10, 43A77, 43A90.

1. Introduction. In this paper, we prove some theorems on absolutely convergent Fourier series in the metric space $L_{2}(G)$, where $G$ is a compact group. The algebra of absolutely convergent Fourier series is a subject matter about which a good deal, although far from everything, is known (see [5, page 328]). Like many branches of harmonic analysis on $T$ and $R$, the theory of absolutely convergent Fourier series is a fruitful source of questions about the corresponding entity for compact groups. By using some absolute convergence theorems of the classical Fourier series, (see [1, 11]), a generalized form of Stechkin [6] and Szasz theorem [1, 11] of the Fourier series on compact groups is obtained. Thus, we solve open problems formulated in [5, page 366] (see also [3, Chapter I, page 9]).

2. Preliminaries and notation. Now, we explain some of the notation and terminologies used throughout the paper.

Let $G$ be a compact group with a dual space $\hat{G}, d g$ denote the Haar measure on $G$ normalized by the condition $\int_{G} d g=1$, and $\int_{G} f(g) d g$ denote the Haar integral of a function $f$ on $G$. Let $U_{\alpha}, \alpha \in \hat{G}$ denotes the irreducible unitary representation of $G$ in the finite dimensional Hilbert space $V_{\alpha}$. We reserve the symbol $d_{\alpha}$ for the dimension of $U_{\alpha}$. Thus, $d_{\alpha}$ is a positive integer. Also, we denote by $\chi_{\alpha}$ and $t_{i j}^{\alpha}\left(i, j=1,2, \ldots, d_{\alpha}\right), \alpha \in \hat{G}$ the character and matrix elements (coordinate functions) of $U_{\alpha}$, respectively.

Let $L_{p}(G)$ be the space of all functions $f$ equipped with the norm

$$
\|f\|_{p}=\left\{\int_{G}|f(g)|^{p} d g\right\}^{1 / p} .
$$

We write $\|\cdot\|_{p}$ instead of $\|\cdot\|_{L_{p}(G)}$, and $L_{\infty}=C$ is the corresponding space of continuous functions, and $\|f\|=\max \{|f(g)|: g \in G\}$. As it is known (see [4] 
or [10, page 99]), the space $L_{2}(G)$ can be decomposed into the sum

$$
L_{2}(G)=\sum_{\alpha \in \hat{G}} \oplus H_{\alpha}
$$

where

$$
H_{\alpha}=\left\{f \in C(G): f(g)=\operatorname{tr}\left(U_{\alpha}(g) C\right), C=\operatorname{Hom}\left(V_{\alpha}, V_{\alpha}\right)\right\}
$$

This theorem is one of the most important results of the harmonic analysis on compact groups. The orthogonal projection $Y_{\alpha}: L_{2}(G) \rightarrow H_{\alpha}$ is given by

$$
\left(Y_{\alpha} f\right)(g)=d_{\alpha} \int_{G} f(h) \chi_{\alpha}\left(g h^{-1}\right) d h
$$

where $\left(Y_{\alpha} f\right)(g)$ does not depend on the choice of a basis in $L_{2}$. Carrying out this construction for every space $H_{\alpha}, \alpha \in \hat{G}$, we obtain an orthonormal basis in $L_{2}$ consisting of the functions $\sqrt{d_{\alpha}} t_{i j}^{\alpha}, \alpha \in \hat{G}, 1 \leq i, j \leq d_{\alpha}$. Any function $f \in L_{2}(G)$ can be expanded into a Fourier series with respect to this basis

$$
f(g)=\sum_{\alpha \in \hat{G}} \sum_{i, j=1}^{d_{\alpha}} a_{i j}^{\alpha} t_{i j}^{\alpha}(g),
$$

where the Fourier coefficients $a_{i j}^{\alpha}$ are defined by the following relations:

$$
a_{i j}^{\alpha}=d_{\alpha} \int_{G} f(g) \overline{t_{i j}^{\alpha}(g)} d g
$$

such that $\overline{t_{i j}^{\alpha}(g)}=t_{i j}^{\alpha}\left(g^{-1}\right)$, where $g^{-1}$ is the inverse of $g$. Note that (2.5) is a convergent series in the mean and that the Parseval's equality

$$
\int_{G}|f(g)|^{2} d g=\sum_{\alpha \in \hat{G}} \frac{1}{d_{\alpha}} \sum_{i, j=1}^{d_{\alpha}}\left|a_{i j}^{\alpha}\right|^{2}
$$

holds. The aforementioned result of harmonic analysis on a compact group can be found, for example, in $[4,5,7,10]$.

We denote by $\mathrm{Sh}_{u}$ the generalized translation operator on compact group $G$ defined by

$$
\begin{gathered}
\left(\mathrm{Sh}_{u} f\right)(g)=\int_{G} f\left(t u t^{-1} g\right) d t \\
\left(\triangle_{u} f\right)(g)=f(g)-\left(\operatorname{Sh}_{u} f\right)(g)=\left(E-\mathrm{Sh}_{u}\right) f
\end{gathered}
$$


where $u, g \in G$ and $E$ is the identity operator. We set

$$
\triangle_{u}^{k} f=\triangle_{u}\left(\triangle_{u}^{k-1} f\right)=\left(E-\operatorname{Sh}_{u}\right)^{k} f=\sum_{i=0}^{k}(-1)^{k+i} C_{k}^{i} \operatorname{Sh}_{u}^{i} f
$$

in which $\operatorname{Sh}_{u}^{0} f=f$ and $\operatorname{Sh}_{u}\left(\operatorname{Sh}_{u}^{i-1} f\right)=\operatorname{Sh}_{u}^{i} f, i=1,2, \ldots, k$ and $k \in N$.

We note that $\alpha$ is a complicated index. Since $\hat{G}$ is a countable set, there are only countably many $\alpha \in \hat{G}$ for which $\alpha_{i j}^{\alpha} \neq 0$ for some $i$ and $j$; enumerate them as $\left\{\alpha_{0}, \alpha_{1}, \ldots, \alpha_{n}, \ldots\right\}$. So, $d_{\alpha_{0}}<d_{\alpha_{1}}<d_{\alpha_{2}}<\cdots<d_{\alpha_{n}}<\cdots$. Because of that, the symbol " $\alpha<n$ " is interpreted as $\left\{\alpha_{0}, \alpha_{1}, \ldots, \alpha_{n-1}\right\} \subset \hat{G}$, and $\alpha \geq n$ denotes the set $\hat{G} \backslash(\alpha<n)$. Let $d_{\alpha}$, as usual, be the dimension of $U_{\alpha}$. For typographical convenience, we write $d_{n}$ for the dimension of the representation $U^{\alpha_{n}}, n=$ $1,2, \ldots$ (See [5, page 458$]$.)

We denote by $E_{n}(f)_{p}$ the approximation of the function $f \in L_{p}(G)$ by "Spherical" polynomials of degree not greater than $n$ :

$$
E_{n}(f)_{p}=\inf \left\{\left\|f-T_{n}\right\|_{p}: T_{n} \in \sum_{\alpha<n, \alpha \in \hat{G}} \oplus H_{\alpha}\right\}
$$

The sequence of best approximations $\left\{E_{n}(f)_{p}\right\}_{n=0}^{\infty}$ is a constructive characteristic of the function $f$. In the capacity of structural characteristic of the function $f$ on a compact group $G$, we define its Spherical modulus of smoothness of order $k$ by

$$
\omega_{k}(f ; \tau)_{p}=\sup \left\{\left\|\left(E-\operatorname{Sh}_{u}\right)^{k} f\right\|_{p}: u \in W_{\tau}\right\}
$$

where $W_{\tau}$ is a neighborhood of $e$ in $G$. In other words,

$$
W_{\tau}=\{u: \rho(u, e)<\tau, u \in G\},
$$

where $\rho$ is a pseudometric on $G$ and $\tau$ is any positive real number. It is easy to show the following properties of $\omega_{k}(f, \tau)_{p}$ :

(a) $\lim _{\tau \rightarrow 0} \omega_{k}(f, \tau)_{p}=0$;

(b) $\omega_{k}(f, \tau)_{p}$ is a continuous monotonically increasing function with respect to $T$;

(c) $\omega_{k}\left(f_{1}+f_{2}, \tau\right)_{p} \leq \omega_{k}\left(f_{1}, \tau\right)_{p}+\omega_{k}\left(f_{2}, \tau\right)_{p}$;

(d) $\omega_{k+l}(f, \tau)_{p} \leq 2^{l} \omega_{k}(f, \tau)_{p}, l=1,2, \ldots$

3. Main results. We need the following simple but useful lemma. 
LEMMA 3.1. The following equality holds for all $u, g \in G$ :

$$
\left(\operatorname{Sh}_{u} t_{i j}^{\alpha}\right)(g)=\frac{\chi_{\alpha}(u)}{d_{\alpha}} t_{i j}^{\alpha}(g) .
$$

PROoF. Using the orthogonality relations and other formulas for matrix elements $t_{i j}^{\alpha}(g)$ (see [7, page 189]), we have

$$
\begin{aligned}
\int_{G} t_{i j}^{\alpha}\left(t u t^{-1} g\right) d t & =\sum_{p=1}^{d_{\alpha}} \sum_{q=1}^{d_{\alpha}} t_{q p}^{\alpha}(u) t_{i j}^{\alpha}(g) \int_{G} t_{i q}^{\alpha}(t) \overline{t_{q p}^{\alpha}(t)} d t \\
& =\frac{1}{d_{\alpha}} \sum_{p=1}^{d_{\alpha}} t_{p p}^{\alpha}(u) t_{i j}^{\alpha}(g)=\frac{1}{d_{\alpha}} \chi_{\alpha}(u) t_{i j}^{\alpha}(g) .
\end{aligned}
$$

This proves the lemma.

The following formula is the particular event of the above lemma:

$$
\int_{G} \chi_{\alpha}\left(t u t^{-1} g\right) d t=\frac{\chi_{\alpha}(u) \chi_{\alpha}(g)}{d_{\alpha}} .
$$

It can be called a Weyl formula.

We note that the expansion (2.5) is connected with the expansion

$$
f(g)=\sum_{\alpha \in G} Y_{\alpha}(f)(g), \quad Y_{\alpha} \in H_{\alpha},
$$

which is defined by (2.4), that is, by the equality

$$
Y_{\alpha}(f)(g)=\sum_{i, j=1}^{d_{\alpha}} a_{i j}^{\alpha} t_{i j}^{\alpha}(g) .
$$

Thus, the coefficients $a_{i j}^{\alpha}$ are defined by (2.6). Using Lemma 3.1 and the definition of $Y_{\alpha}$, we obtain

$$
\begin{aligned}
Y_{\alpha}\left(\operatorname{Sh}_{u} f\right)(g) & =\sum_{i, j=1}^{d_{\alpha}} a_{i j}^{\alpha} \int_{G} t_{i j}^{\alpha}\left(t u t^{-1} g\right) d t \\
& =\sum_{i, j=1}^{d_{\alpha}} a_{i j}^{\alpha} \frac{\chi_{\alpha}(u)}{d_{\alpha}} t_{i j}^{\alpha}(g) \\
& =\frac{\chi_{\alpha}(u)}{d_{\alpha}} Y_{\alpha}(f)(g) .
\end{aligned}
$$

The following are simple facts with frequent usage: if $f \in L_{p}$, then

(1) $\left\|\mathrm{Sh}_{u} f\right\|_{p} \leq\|f\|_{p}$;

(2) $\left\|f-\mathrm{Sh}_{u} f\right\|_{p} \rightarrow 0$ as $u \rightarrow e$;

(3) $\left(Y_{\alpha}\left(\operatorname{Sh}_{u} f\right)\right)(g)=\left(\chi_{\alpha}(u) / \chi_{\alpha}(e)\right)\left(Y_{\alpha} f\right)(g)$ for all $\alpha \in \hat{G}$.

We note that $\chi_{\alpha}(e)=d_{\alpha}$. 
THEOREM 3.2. If $f \in L_{2}$ and $f$ is not constant, then

$$
E_{n}(f)_{2} \leq \sqrt{\frac{d_{n}}{d_{n}-2 k}} \omega_{k}\left(f ; \frac{1}{n}\right)_{2}, \quad n=1,2, \ldots
$$

Proof. Let $f \in L_{2}$ and $S_{n}(f, g)$ denote the $n$th partial sum of the Fourier series (2.5), that is,

$$
S_{n}(f, g)=\sum_{\alpha<n} \sum_{i, j=1}^{d_{\alpha}} a_{i j}^{\alpha} t_{i j}^{\alpha}(g)=\sum_{p=0}^{n} \sum_{i, j=1}^{d_{\alpha_{p}}} a_{i j}^{\alpha_{p}} t_{i j}^{\alpha_{p}}(g) .
$$

Using Parseval's equality for the compact group $G$, we have

$$
E_{n}^{2}(f)_{2}=\left\|f-S_{n}(f)\right\|_{2}^{2}=\sum_{\alpha \geq n} \frac{1}{d_{\alpha}} \sum_{i, j=1}^{d_{\alpha}}\left|a_{i j}^{\alpha}\right|^{2} .
$$

Using (3), it is not hard to see that

$$
\left(Y_{\alpha}\left(\triangle^{k} f\right)\right)(g)=\left(1-\frac{\chi_{\alpha}(u)}{d_{\alpha}}\right)^{k}\left(Y_{\alpha} f\right)(g), \quad \alpha \in \hat{G}
$$

Consequently, $\left(\triangle^{k} f\right)(g)=\sum_{\alpha \in \hat{G}}\left(1-\chi_{\alpha}(u) / d_{\alpha}\right)^{k} a_{i j}^{\alpha} t_{i j}^{\alpha}$. By another application of Parseval's equality, we obtain

$$
\begin{aligned}
\left\|\triangle_{u}^{k} f\right\|_{2}^{2} & =\sum_{\alpha \in \hat{G}} \frac{1}{d_{\alpha}} \sum_{i, j=1}^{d_{\alpha}}\left|1-\frac{\chi_{\alpha}(u)}{d_{\alpha}}\right|^{2 k}\left|a_{i j}^{\alpha}\right|^{2} \geq \sum_{\alpha \geq n} \frac{1}{d_{\alpha}} \sum_{i, j=1}^{d_{\alpha}}\left|1-\frac{\chi_{\alpha}(u)}{d_{\alpha}}\right|^{2 k}\left|a_{i j}^{\alpha}\right|^{2} \\
& =\sum_{\alpha \geq n} \frac{1}{d_{\alpha}} \sum_{i, j=1}^{d_{\alpha}}\left(1-\frac{2 \operatorname{Re} \chi_{\alpha}(u)}{d_{\alpha}}+\frac{\left|\chi_{\alpha}(u)\right|^{2}}{d_{\alpha}^{2}}\right)^{k}\left|a_{i j}^{\alpha}\right|^{2} .
\end{aligned}
$$

Now, using Bernolly's inequality $(1+x)^{k} \geq 1+k x$ for $x \geq-1$, we obtain

$$
\left\|\triangle_{u}^{k} f\right\|_{2}^{2} \geq \sum_{\alpha \geq n} \frac{1}{d_{\alpha}} \sum_{i, j=1}^{d_{\alpha}}\left(1-\frac{2 k \operatorname{Re} \chi_{\alpha}(u)}{d_{\alpha}}+\frac{k\left|\chi_{\alpha}(u)\right|^{2}}{d_{\alpha}^{2}}\right)\left|a_{i j}^{\alpha}\right|^{2} .
$$

Consequently,

$$
\left\|\triangle_{u}^{k} f\right\|_{2}^{2} \geq \sum_{\alpha \geq n} \frac{1}{d_{\alpha}} \sum_{i, j=1}^{d_{\alpha}}\left|a_{i j}^{\alpha}\right|^{2}-\sum_{\alpha \geq n} \frac{1}{d_{\alpha}} \sum_{i, j=1}^{d_{\alpha}} \frac{2 k \operatorname{Re} \chi_{\alpha}(u)}{d_{\alpha}}\left|a_{i j}^{\alpha}\right|^{2}
$$

therefore,

$$
E_{n}^{2}(f)_{2} \leq\left\|\triangle_{u}^{k} f\right\|_{2}^{2}+2 k \sum_{\alpha \geq n} \frac{1}{d_{\alpha}} \sum_{i, j=1}^{d_{\alpha}} \frac{\operatorname{Re} \chi_{\alpha}(u)}{d_{\alpha}}\left|a_{i j}^{\alpha}\right|^{2}
$$


Let $\Phi_{W_{\tau}}$ be a nonnegative integrable function vanishing outside $W_{T}$ and satisfying the condition $\int_{G} \Phi_{W_{\tau}}(g) d g=1$. For example, we can take $\Phi_{W_{\tau}}=\xi_{W_{\tau}} / \mu\left(W_{\tau}\right)$, where $\mu\left(W_{\tau}\right)$ is the Haar measure of $W_{\tau}$ and $\xi_{W_{\tau}}$ is the characteristic function of $W_{\tau}$. Multiplying both sides of (3.14) by $\Phi_{W_{1 / n}}$, and integrating with respect to $u$ on $G$, and using the equality $\int_{G}\left|\chi_{\alpha}\right|^{2} d g=1$ (see [7, page 195]), we obtain

$$
\begin{aligned}
\int_{G} E_{n}^{2}(f)_{2} \Phi_{W_{1 / n}}(u) d u \leq & \int_{G}\left\|\triangle_{u}^{k} f\right\|_{2}^{2} \Phi_{W_{1 / n}} d u \\
& +2 k \sum_{\alpha \geq n} \frac{1}{d_{\alpha}^{2}} \sum_{i, j=1}^{d_{\alpha}}\left|a_{i j}^{\alpha}\right|^{2} \int_{G}\left|\chi_{\alpha}(u)\right| \Phi_{W_{1 / n}}(u) d u \\
\leq & \sup \left\|\triangle_{u}^{k} f\right\|_{2}^{2}+\frac{2 k}{d_{n}} \sum_{\alpha \geq n} \frac{1}{d_{\alpha}} \sum_{i, j=1}^{d_{\alpha}}\left|a_{i j}^{\alpha}\right|^{2} .
\end{aligned}
$$

Therefore, it is not hard to see that

$$
E_{n}^{2}(f)_{2} \leq \omega_{k}^{2}\left(f, \frac{1}{n}\right)_{2}+\frac{2 k}{d_{n}} E_{n}^{2}(f)_{2} .
$$

Finally, we obtain

$$
E_{n}(f)_{2} \leq \sqrt{\frac{d_{n}}{d_{n}-2 k}} \omega_{k}\left(f, \frac{1}{n}\right)_{2},
$$

which proves the theorem.

This theorem is given without proof in [8] for the case where $k=1$.

We note that the matrix elements of unitary representations $t_{i j}^{\alpha}(g)$ satisfy the relations

$$
\sum_{j=1}^{d_{\alpha}} t_{i j}^{\alpha}(g) \overline{t_{k j}^{\alpha}(g)}=\sum_{j=1}^{d_{\alpha}^{\alpha}} t_{i j}^{\alpha}(g) \overline{t_{j k}^{\alpha}(g)}= \begin{cases}0 & \text { if } i \neq k, \\ 1 & \text { if } i=k .\end{cases}
$$

In particular, we have

$$
\sum_{j=1}^{d_{\alpha}}\left|t_{i j}^{\alpha}\right|^{2}=1 \Longrightarrow\left|t_{i j}^{\alpha}(g)\right| \leq 1
$$

for all $\alpha \in \hat{G}$ and $i, j=1,2, \ldots, d_{\alpha}$. Furthermore, it is obvious that $\left|a_{i j}^{\alpha} t_{i j}^{\alpha}(g)\right| \leq$ $\left|a_{i j}^{\alpha}\right|$; therefore, according to the sufficient condition for absolutely convergent Fourier series on the group $G$, the series $\sum_{\alpha \in \hat{G}} \sum_{i, j=1}^{\alpha}\left|a_{i j}^{\alpha}\right|$ is convergent. Let $A(G):=\left\{f: \sum_{\alpha \in \hat{G}} \sum_{i, j=1}^{\alpha}\left|a_{i j}^{\alpha}\right|<+\infty\right\}$. Using Theorem 3.2, and repeating the proof of analogous theorems (see [1, Chapter IX] or [6, Chapter II]) with some changes, we obtain the following theorems. 
THEOREM 3.3. If $f(g) \in L_{2}(G)$, then

$$
\sum_{n=1}^{\infty} \frac{\omega_{k}(f, 1 / n)_{2}}{\sqrt{n}}<+\infty \Longrightarrow f(g) \in A(G) .
$$

This theorem is analogous to the Szasz theorem of the classical Fourier series in the case where $k=1$ and $G=T$.

THEOREM 3.4. If $f(g) \in L_{2}(G)$, then

$$
\sum_{n=1}^{\infty} \frac{E_{n}(f)_{2}}{\sqrt{n}}<+\infty \Longrightarrow f(g) \in A(G)
$$

This theorem is also analogous to a theorem in trigonometric case proved by Stechkin [9].

4. Applications to compact group SU(2). The group SU(2) consists of unimodular unitary matrices of the second order, that is, matrices of the form

$$
u=\left(\begin{array}{cc}
\alpha & \beta \\
-\bar{\beta} & \bar{\alpha}
\end{array}\right), \quad|\alpha|^{2}+|\beta|^{2}=1 .
$$

Therefore, each element $u$ of $\mathrm{SU}(2)$ is uniquely determined by a pair of complex numbers $\alpha$ and $\beta$ such that $|\alpha|^{2}+|\beta|^{2}=1$. We have (see [5]) the relation “ $(\alpha, \beta) \mapsto(\phi, \theta, \psi)$," where $\alpha \beta \neq 0,|\alpha|^{2}+|\beta|^{2}=1$, and the parameters $\phi, \theta$, and $\psi$ are called Euler angles defined by

$$
|\alpha|=\cos \frac{\theta}{2} ; \quad \operatorname{Arg} \alpha=\frac{\phi+\psi}{2} ; \quad \operatorname{Arg} \beta=\frac{\phi-\psi}{2} .
$$

Let $\phi, \theta$, and $\psi$ satisfy the conditions

$$
0 \leq \phi<2 \pi, \quad 0 \leq \theta<\pi, \quad-2 \pi \leq \psi<2 \pi .
$$

Also, we know that the dimension of the representation $T^{l}$ of SU(2) is equal to $2 l+1$, where $l=0,1 / 2,1, \ldots$ and the matrix elements of $T^{l}$ for group SU(2) are defined by

$$
t_{m n}^{l}(u)=e^{-(n \psi+m \phi)} P_{m n}^{l}(\cos \theta) i^{(m-n)}
$$

Expressing $t_{m n}^{l}(u)$ in terms of $P_{m n}^{l}(\cos \theta)$, we arrive at the following conclusion:

Any function $f(\phi, \theta, \psi), 0 \leq \phi<2 \pi, 0 \leq \theta<\pi$, and $-2 \pi \leq \psi<2 \pi$ belonging to the space $L^{2}(\mathrm{SU}(2))$ such that

$$
\int_{-2 \pi}^{2 \pi} \int_{0}^{2 \pi} \int_{0}^{\pi}|f(\phi, \theta, \psi)|^{2} \sin \theta d \theta d \phi d \psi<\infty
$$


can be expanded into the mean-convergent series

$$
f(\phi, \theta, \psi)=\sum_{l} \sum_{m=-l}^{l} \sum_{n=-l}^{l} \alpha_{m n}^{l} e^{-i(m \phi+n \psi)} P_{m n}^{l}(\cos \theta),
$$

where

$$
\alpha_{m n}^{l}=\frac{2 l+1}{16 \pi^{2}} \int_{-2 \pi}^{2 \pi} \int_{0}^{2 \pi} \int_{0}^{\pi} f(\phi, \theta, \psi) e^{i(m \phi+n \psi)} P_{m n}^{l}(\cos \theta) \sin \theta d \theta d \phi d \psi .
$$

In addition, we obtain from Parseval's equality that

$$
\sum_{l} \sum_{m=-l}^{l} \sum_{n=-l}^{l} \frac{1}{2 l+1}\left|\alpha_{m n}^{l}\right|^{2}=\frac{1}{16 \pi^{2}} \int_{-2 \pi}^{2 \pi} \int_{0}^{2 \pi} \int_{0}^{\pi}|f(\phi, \theta, \psi)|^{2} \sin \theta d \theta d \phi d \psi .
$$

Using Theorem 3.2, we obtain the following theorem.

THEOREM 4.1. If $f(\phi, \theta, \psi) \in L_{2}(\mathrm{SU}(2))$, then

$$
\begin{gathered}
E_{n}(f)_{2} \leq \sqrt{1+\frac{2}{n-1}} \omega_{k}\left(f, \frac{1}{n}\right)_{2}, \\
\left\{\sum_{l \geq n} \sum_{m=-l}^{l} \sum_{n=-l}^{l} \frac{1}{2 l+1}\left|\alpha_{m n}^{l}\right|^{2}\right\}^{1 / 2} \leq \sqrt{1+\frac{2}{n-1}} \omega_{k}\left(f, \frac{1}{n}\right)_{2} .
\end{gathered}
$$

Using the relation between the polynomial $P_{n}^{(\alpha, \beta)}(z)$ and $P_{m n}^{l}(z)$, we conclude that

$$
P_{m n}^{l}(z)=2^{-m}\left[\frac{(l-m) !(l+m) !}{(l-n) !(l+n) !}\right]^{1 / 2}(1-z)^{(m-n) / 2}(1+z)^{(m+n) / 2} P_{l-m}^{(m-n, m+n)}
$$

The Jacobi polynomials obtained here are characterized by the condition that $\alpha$ and $\beta$ are integers and $n+\alpha+\beta \in Z_{+}$.

Now, we consider the following case.

Let $L_{2}^{(\alpha, \beta)}[-1,1]$ be the Hilbert space of the functions $f$ defined on the segment $[-1,1]$ with the scalar product

$$
\left(f_{1}, f_{2}\right)=\int_{-1}^{1} f_{1}(x) \overline{f_{2}(x)}(1-x)^{\alpha}(1+x)^{\beta} d x
$$

then, any function $f$ in this space is expanded into the mean-convergent series

$$
f(x)=\sum_{n=0}^{\infty} \alpha_{n} \hat{P}_{n}^{(\alpha, \beta)}(x)
$$


where the polynomials $\hat{P}_{n}^{(\alpha, \beta)}(x)$ are given by

$$
\begin{gathered}
\hat{P}_{k}^{(\alpha, \beta)}(x)=2^{-(\alpha+\beta+1) / 2}\left[\frac{k !(k+\alpha+\beta) !(\alpha+\beta+2 k+1)}{(k+\alpha) !(k+\beta) !}\right]^{1 / 2} P_{k}^{(\alpha, \beta)}(x), \\
\alpha_{n}=\int_{-1}^{1} f(x) \hat{P}_{n}^{(\alpha, \beta)}(x)(1-x)^{\alpha}(1+x)^{\beta} d x .
\end{gathered}
$$

The Parseval's equality

$$
\int_{-1}^{1}|f(x)|^{2}(1-x)^{\alpha}(1+x)^{\beta} d x=\sum_{n=0}^{\infty}|\alpha|^{2}
$$

holds. The formulas (4.12), (4.14), and (4.15) are proved for integral nonnegative values of $\alpha$ and $\beta$. We can show that they are valid for arbitrary real values of $\alpha$ and $\beta$ exceeding -1 . Finally, we reach the following theorem.

THEOREM 4.2. If $f(x) \in L_{2}[-1,1]$, then the following hold for Jacobi series:

$$
\begin{gathered}
E_{n}(f)_{2} \leq \sqrt{1+\frac{2}{n-1}} \omega_{k}\left(f, \frac{1}{n}\right)_{2}, \\
\left\{\sum_{l=n}^{\infty}\left|\alpha_{l}\right|^{2}\right\}^{1 / 2} \leq \sqrt{1+\frac{2}{n-1}} \omega_{k}\left(f, \frac{1}{n}\right)_{2} .
\end{gathered}
$$

NoTE. For the ideas similar to this paper we refer to [2] and its references.

ACKNOWLEDGMENTS. This research was supported by Tabriz University. We would like to thank the research office of Tabriz University for its support.

\section{REFERENCES}

[1] W. K. Bari, Trigonometric Series, vol. II, Holt, Rinehart and Winston, New York, 1967.

[2] G. Benke, Bernštěn's theorem for compact groups, J. Funct. Anal. 35 (1980), no. 3, 295-303.

[3] R. E. Edwards, Fourier series. A Modern Introduction. Vol. 1, 2nd ed., Graduate Texts in Mathematics, vol. 64, Springer-Verlag, New York, 1979.

[4] S. Helgason, Groups and Geometric Analysis, Pure and Applied Mathematics, vol. 113, Academic Press, Florida, 1984.

[5] E. Hewitt and K. A. Ross, Abstract Harmonic Analysis. Vol. II: Structure and Analysis for Compact Groups. Analysis on Locally Compact Abelian Groups, Die Grundlehren der Mathematischen Wissenschaften, vol. 152, SpringerVerlag, New York, 1970 (German).

[6] J.-P. Kahane, Séries de Fourier absolument convergentes, Ergebnisse der Mathematik und ihrer Grenzgebiete, vol. 50, Springer-Verlag, Berlin, 1970 (French).

[7] M. A. Naĭmark and A. I. Štern, Theory of Group Representations, Grundlehren der Mathematischen Wissenschaften, vol. 246, Springer-Verlag, New York, 1982 (German). 
[8] S. F. Rzaev, L2-Approximation on compact groups, Proc. "Questions on Functional Analysis and Mathematical Physics Conference", Baku, 1999, pp. 418-419.

[9] S. B. Stechkin, On absolute convergence of orthogonal series, Dokl. Akad. Nauk. SSSR 102 (1955), 37-40.

[10] N. Ja. Vilenkin and A. U. Klimyk, Representation of Lie groups and Special Functions. Vol. 1, Mathematics and Its Applications, vol. 72, Kluwer Academic Publishers, Dordrecht, 1991.

[11] A. Zygmund, Trigonometric Series. 2nd ed. Vols. I, II, Cambridge University Press, New York, 1959.

H. Vaezi: Faculty of Mathematical Sciences, University of Tabriz, Tabriz, Iran E-mail address: hvaezi@tabrizu.ac.ir

S. F. Rzaev: Institute of Mathematics and Mechanics, Azerbaijan Academy of Sciences, Baku, Azerbaijan

E-mail address: rzseymur@hotmai 1.com 


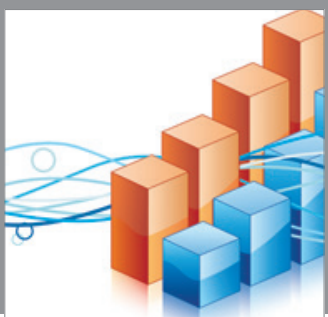

Advances in

Operations Research

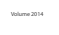

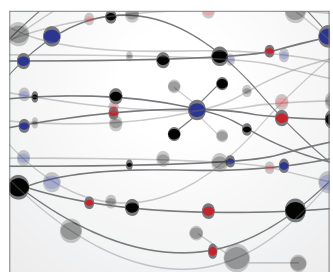

\section{The Scientific} World Journal
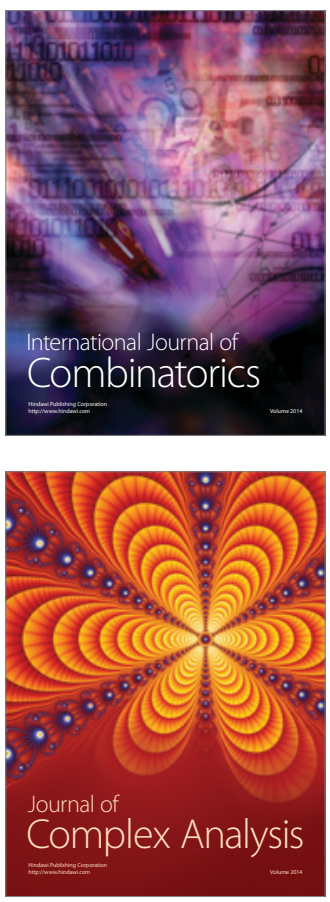

International Journal of

Mathematics and

Mathematical

Sciences
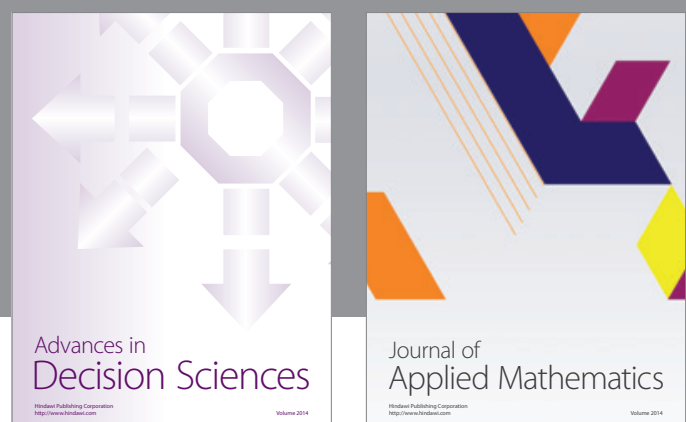

Journal of

Applied Mathematics
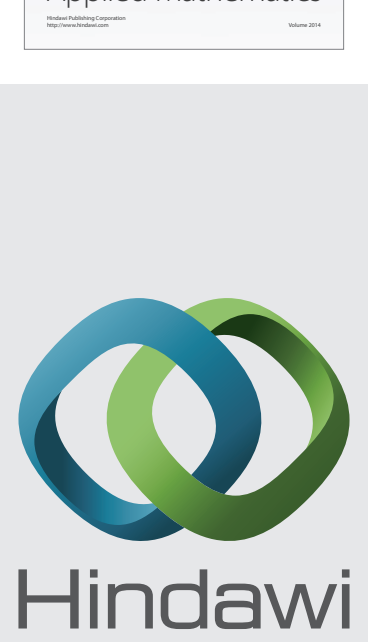

Submit your manuscripts at http://www.hindawi.com
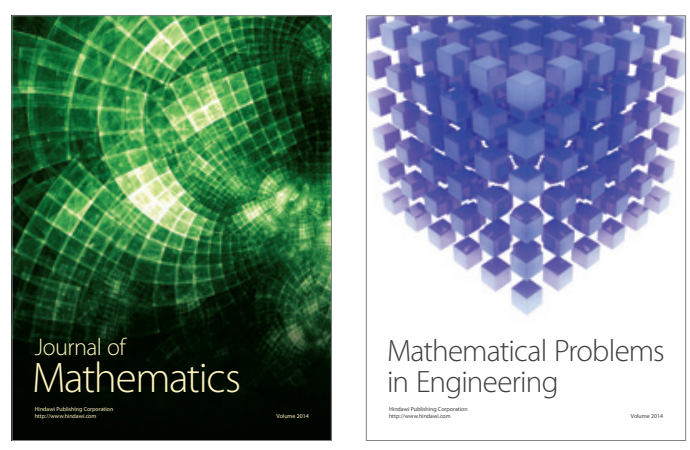

Mathematical Problems in Engineering
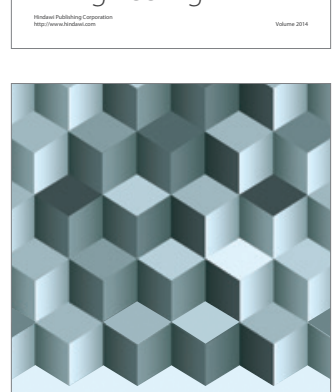

Journal of

Function Spaces
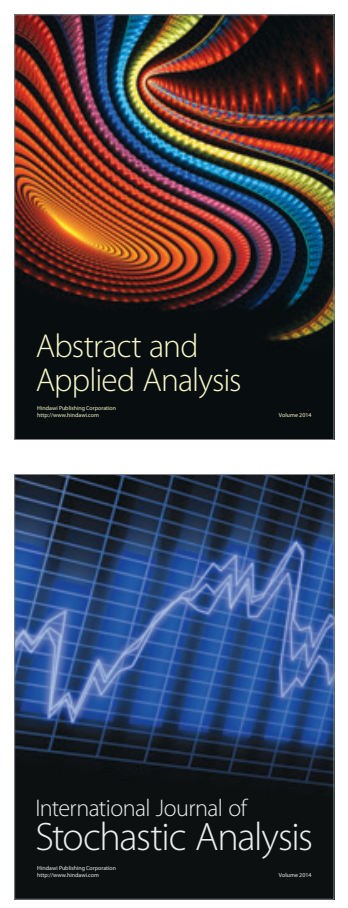

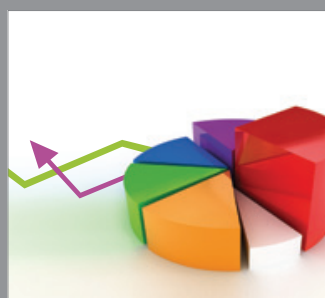

ournal of

Probability and Statistics

Promensencen
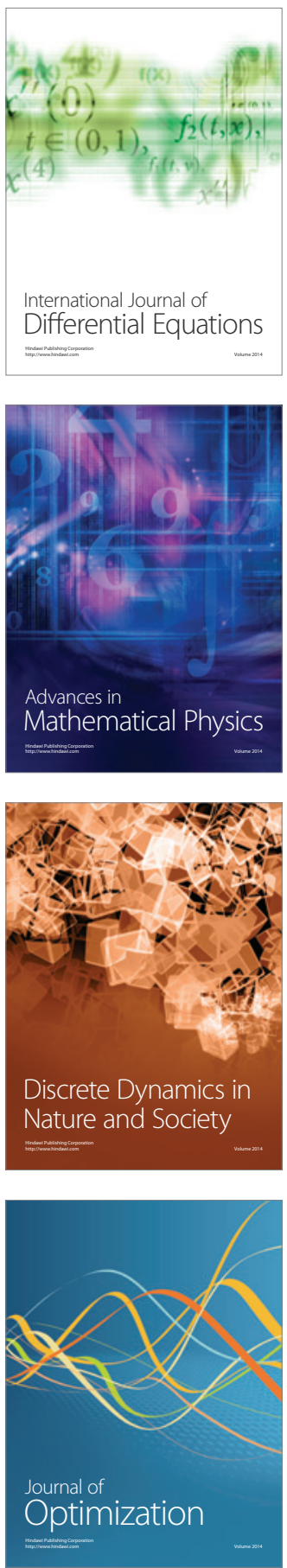